\title{
Deciding Full Branching Time Logic by Program Transformation
}

\author{
Alberto Pettorossi ${ }^{1}$, Maurizio Proietti ${ }^{2}$, and Valerio Senni ${ }^{1}$ \\ 1 DISP, University of Rome Tor Vergata, Via del Politecnico 1, I-00133 Rome, Italy \\ \{pettorossi, senni\}@disp.uniroma2.it \\ 2 IASI-CNR, Viale Manzoni 30, I-00185 Rome, Italy \\ proietti@iasi.cnr.it
}

\begin{abstract}
We present a method based on logic program transformation, for verifying Computation Tree Logic $\left(\mathrm{CTL}^{*}\right)$ properties of finite state reactive systems. The finite state systems and the CTL* properties we want to verify, are encoded as logic programs on infinite lists. Our verification method consists of two steps. In the first step we transform the logic program that encodes the given system and the given property, into a monadic $\omega$-program, that is, a stratified program defining nullary or unary predicates on infinite lists. This transformation is performed by applying unfold/fold rules that preserve the perfect model of the initial program. In the second step we verify the property of interest by using a proof method for monadic $\omega$-programs.
\end{abstract}

\section{Introduction}

The branching time temporal logic $\mathrm{CTL}^{*}$ is among the most popular temporal logics that have been proposed for verifying properties of reactive systems [4]. A finite state reactive system, such as a protocol, a concurrent system, or a digital circuit, is formally specified as a Kripke structure and the property to be verified is specified as a CTL* formula. Thus, the problem of checking whether or not a reactive system satisfies a given property is reduced to the problem of checking whether or not a Kripke structure is a model of a CTL* formula.

There is a vast literature on the problem of model checking for the CTL* logic and, in particular, its two fragments: (i) the Computational Tree Logic CTL, and (ii) the Linear-time Temporal Logic LTL (see [2] for a survey). Most of the known model checking algorithms for CTL* either combine model checking algorithms for CTL and LTL [2], or use techniques based on translations to automata on infinite trees [6].

In this paper we extend to $\mathrm{CTL}^{*}$ a method proposed in [11] for LTL. We encode the satisfaction relation of a $\mathrm{CTL}^{*}$ formula $\varphi$ with respect to a Kripke structure $\mathcal{K}$ by means of a locally stratified logic program $P_{\mathcal{K}, \varphi}$. The program $P_{\mathcal{K}, \varphi}$ belongs to a class of programs, called $\omega$-programs, which define predicates on infinite lists. Predicates of this type are needed because the definition of the satisfaction relation is based on the infinite computation paths of $\mathcal{K}$. The semantics of $P_{\mathcal{K}, \varphi}$ is provided by its unique perfect model [12] which for $\omega$-programs is defined in terms of a non-Herbrand interpretation for infinite lists. 
Our verification method consists of two steps. In the first step we transform the program $P_{\mathcal{K}, \varphi}$ into a monadic $\omega$-program, that is, a stratified program that defines nullary or unary predicates on infinite lists. This transformation is performed by applying unfold/fold transformation rules similar to those presented in $[5,14,15]$ according to a strategy which is a variant of the specialization strategy presented in [5]. Similarly to $[5,14]$, the use of those unfold/fold rules guarantees the preservation of the perfect model of $P_{\mathcal{K}, \varphi}$.

In the second step of our verification method we apply a proof method for monadic $\omega$-programs which is sound and complete with respect to the perfect model semantics.

The paper is structured as follows. In Section 2 we introduce the class of $\omega$-programs and we show how to encode the satisfaction relation for any given Kripke structure and CTL* formula as an $\omega$-program. In Section 3 we present our verification method. In particular, in Section 3.1 we present the specialization strategy for transforming an $\omega$-program into a monadic $\omega$-program and in Section 3.2 we present the proof method for monadic $\omega$-programs. Finally, in Section 4 we discuss related work in the area of model checking and logic programming.

\section{Encoding CTL* Model Checking as a Logic Program}

In this section we describe a method which, given a Kripke structure $\mathcal{K}$ and a CTL* state formula $\varphi$, allows us to construct a logic program $P_{\mathcal{K}, \varphi}$ and to define a nullary predicate prop such that $\varphi$ is true in $\mathcal{K}$, written $\mathcal{K} \vDash \varphi$, iff prop is true in the perfect model of $P_{\mathcal{K}, \varphi}$, written $M\left(P_{\mathcal{K}, \varphi}\right) \vDash$ prop. Thus, the problem of checking whether or not $\mathcal{K} \vDash \varphi$ holds, also called the problem of model checking $\varphi$ with respect to $\mathcal{K}$, is reduced to the problem of testing whether or not $M\left(P_{\mathcal{K}, \varphi}\right) \vDash$ prop holds.

Now we briefly recall the definition of the temporal logic $\mathrm{CTL}^{*}$ (see [2] for more details). A Kripke structure is a 4 -tuple $\left\langle\Sigma, s_{0}, \rho, \lambda\right\rangle$, where: (i) $\Sigma=$ $\left\{s_{0}, \ldots, s_{h}\right\}$ is a finite set of states, (ii) $s_{0} \in \Sigma$ is the initial state, (iii) $\rho \subseteq \Sigma \times \Sigma$ is a total transition relation, and (iv) $\lambda: \Sigma \rightarrow \mathcal{P}($ Elem) is a total function that assigns to every state $s \in \Sigma$ a subset $\lambda(s)$ of the set Elem of elementary properties. A computation path of $\mathcal{K}$ from a state $s$ is an infinite list $\left[a_{0}, a_{1}, \ldots\right]$ of states such that $a_{0}=s$ and, for every $i \geq 0,\left(a_{i}, a_{i+1}\right) \in \rho$. Given an infinite list $\pi=\left[a_{0}, a_{1}, \ldots\right]$ of states, by $\pi_{j}$, for any $j \geq 0$, we denote the infinite list which is the suffix $\left[a_{j}, a_{j+1}, \ldots\right]$ of $\pi$.

Definition 1 (CTL* Formulas). Given a set Elem of elementary properties, a CTL* formula $\varphi$ is either a path formula $\varphi_{p}$ or a state formula $\varphi_{s}$ defined as follows:

(path formulas) $\varphi_{p}::=\varphi_{s}\left|\neg \varphi_{p}\right| \varphi_{p} \wedge \varphi_{p}\left|\mathrm{X} \varphi_{p}\right| \varphi_{p} \mathrm{U} \varphi_{p}$

(state formulas) $\varphi_{s}::=d\left|\neg \varphi_{s}\right| \varphi_{s} \wedge \varphi_{s} \mid \mathrm{E} \varphi_{p}$

where $d \in$ Elem. 
As the following definition formally specifies, (i) $\mathrm{X} \varphi$ holds on a computation path $\pi$ if $\varphi$ holds in the second state of $\pi$, (ii) $\varphi_{1} \mathrm{U} \varphi_{2}$ holds on a computation path $\pi$ if $\varphi_{2}$ holds in a state $s$ of $\pi$ and $\varphi_{1}$ holds in every state preceding $s$ in $\pi$, and (iii) $\mathrm{E} \varphi$ holds in a state $s$ if there exists a computation path starting from $s$ on which $\varphi$ holds.

Definition 2 (Satisfaction Relation for $\mathbf{C T L}^{*}$ ). Let $\mathcal{K}=\left\langle\Sigma, s_{0}, \rho, \lambda\right\rangle$ be a Kripke structure. For any $\mathrm{CTL}^{*}$ formula $\varphi$ and infinite list $\pi \in \Sigma^{\omega}$, the relation $\mathcal{K}, \pi \vDash \varphi$ is inductively defined as follows:

$$
\begin{aligned}
& \mathcal{K}, \pi \vDash d \quad \text { iff } \pi=\left[a_{0}, a_{1}, \ldots\right] \text { and } d \in \lambda\left(a_{0}\right) \\
& \mathcal{K}, \pi \vDash \neg \varphi \quad \text { iff } \mathcal{K}, \pi \not \models \varphi \\
& \mathcal{K}, \pi \vDash \varphi_{1} \wedge \varphi_{2} \text { iff } \mathcal{K}, \pi \vDash \varphi_{1} \text { and } \mathcal{K}, \pi \vDash \varphi_{2} \\
& \mathcal{K}, \pi \vDash \mathrm{X} \varphi \quad \text { iff } \mathcal{K}, \pi_{1} \vDash \varphi \\
& \mathcal{K}, \pi \vDash \varphi_{1} \mathrm{U} \varphi_{2} \text { iff there exists } i \geq 0 \text { such that } \mathcal{K}, \pi_{i} \vDash \varphi_{2} \\
& \text { and, for all } 0 \leq j<i, \mathcal{K}, \pi_{j} \vDash \varphi_{1} \\
& \mathcal{K}, \pi \vDash \mathrm{E} \varphi \quad \text { iff } \pi=\left[a_{0}, a_{1}, \ldots\right] \text { and there exists a computation path } \pi^{\prime} \\
& \text { from } a_{0} \text { such that } \mathcal{K}, \pi^{\prime} \vDash \varphi \text {. }
\end{aligned}
$$

Given a state formula $\varphi$, we say that $\mathcal{K}$ is a model of $\varphi$, written $\mathcal{K} \vDash \varphi$, iff there exists an infinite list $\pi \in \Sigma^{\omega}$ such that the first state of $\pi$ is the initial state $s_{0}$ of $\mathcal{K}$ and $\mathcal{K}, \pi \vDash \varphi$ holds.

The above definition of the satisfaction relation for $\mathrm{CTL}^{*}$ formulas is a shorter, yet equivalent, version of the usual definition one can find in the literature [2].

In order to encode the satisfaction relation for $\mathrm{CTL}^{*}$ formulas as a logic program, we will introduce in the next section a class of logic programs, called $\omega$-programs. In this class the arguments of predicates may denote infinite lists.

\subsection{Syntax and Semantics of $\omega$-Programs}

Let us consider a Kripke structure $\mathcal{K}$. Let us also consider a first order language $\mathcal{L}_{\omega}$ given by a set $\operatorname{Var}$ of variables, a set Fun of function symbols, and a set Pred of predicate symbols. We assume that Fun includes: (i) the set $\Sigma$ of the states of $\mathcal{K}$, each state being a constant of $\mathcal{L}_{\omega}$, (ii) the set Elem of the elementary properties of $\mathcal{K}$, each elementary property being a constant of $\mathcal{L}_{\omega}$, and (iii) the binary function symbol [-|] which is the constructor of infinite lists. Thus, for instance, $[H \mid T]$ is the infinite list whose head is $H$ and whose tail is the infinite list $T$.

We assume that $\mathcal{L}_{\omega}$ is a typed language [9] with the following three basic types: (i) fterm, which is the type of finite terms, (ii) state, which is the type of states, and (iii) ilist, which is the type of infinite lists of states. Every function symbol in Fun $-(\Sigma \cup\{[-\mid-]\})$, with arity $n(\geq 0)$, has type fterm $\times \cdots \times$ fterm $\rightarrow$ fterm, where fterm occurs $n$ times to the left of $\rightarrow$. Every function symbol in $\Sigma$ has arity 0 and type state. The function symbol [-_] has type state $\times$ ilist $\rightarrow$ ilist. A predicate symbol of arity $n(\geq 0)$ in Pred has type of the form $\tau_{1} \times \cdots \times \tau_{n}$, where $\tau_{1}, \ldots, \tau_{n} \in\{$ fterm, state, $i$ ist $\}$. An $\omega$-program is a logic program constructed as usual (see, for instance, [9]) from symbols in the 
typed language $\mathcal{L}_{\omega}$. In what follows, for reasons of simplicity, we will feel free to say 'program', instead of ' $\omega$-program'.

Given a term or a formula $t$, by vars $(t)$ we denote the set of variables occurring in $t$. The same notation will be used for sets of terms and sets of formulas. The existential closure of a formula $\varphi$, denoted $\exists(\varphi)$, is the formula $\exists X_{1} \ldots \exists X_{n} \varphi$ where $\left\{X_{1}, \ldots, X_{n}\right\}$ is the set of the free variables occurring in $\varphi$. The universal closure of a formula $\varphi$, denoted $\forall(\varphi)$, is defined in a similar way by using $\forall$, instead of $\exists$. Note that if $\operatorname{vars}(\varphi)=\emptyset$, then $\exists(\varphi)$ is $\varphi$ itself.

An interpretation for our typed language $\mathcal{L}_{\omega}$, called $\omega$-interpretation, is given as follows. Let $H U$ be the Herbrand universe constructed from the set Fun $-(\Sigma \cup$ $\{[-\mid-]\})$ of function symbols and let $\Sigma^{\omega}$ be the set of the infinite lists of states. An $\omega$-interpretation $I$ is an interpretation such that: (i) $I$ assigns to the types fterm, state, and ilist, respectively, the sets $H U, \Sigma$, and $\Sigma^{\omega}$, (ii) $I$ assigns to the function symbol [-|] the function [-|- $]_{I}$ such that, for any state $a \in \Sigma$ and infinite list $\left[a_{1}, a_{2}, \ldots\right] \in \Sigma^{\omega},\left[a \mid\left[a_{1}, a_{2}, \ldots\right]\right]_{I}$ is the infinite list $\left[a, a_{1}, a_{2}, \ldots\right]$, (iii) $I$ is an Herbrand interpretation for all function symbols in Fun-( $\Sigma \cup\{[-\mid]\})$, and (iv) $I$ assigns to every $n$-ary predicate $p \in$ Pred of type $\tau_{1} \times \ldots \times \tau_{n}$ a relation on $D_{1} \times \cdots \times D_{n}$, where, for $i=1, \ldots, n, D_{i}$ is either $H U$ or $\Sigma$ or $\Sigma^{\omega}$, according to the case where $\tau_{i}$ is either fterm or state or ilist, respectively. We say that an $\omega$-interpretation $I$ is an $\omega$-model of a program $P$ iff for every clause $\gamma \in P$ we have that $I \vDash \forall(\gamma)$.

A level mapping is a function $\ell:$ Pred $\rightarrow \mathbb{N}$. A level mapping is extended to literals as follows: for any literal $L$ having predicate $p$, if $L$ is a positive literal, then $\ell(L)=\ell(p)$ and, if $L$ is a negative literal then $\ell(L)=\ell(p)+1$. An $\omega$ clause $\gamma$ of the form $H \leftarrow L_{1} \wedge \ldots \wedge L_{m}$ is stratified w.r.t. $\ell$ if, for $i=1, \ldots, m$, $\ell(H) \geq \ell\left(L_{i}\right)$. An $\omega$-program $P$ is stratified if there exists a level mapping $\ell$ such that all clauses of $P$ are stratified w.r.t. $\ell$.

A valuation is a function $v: \operatorname{Var} \rightarrow H U \cup \Sigma \cup \Sigma^{\omega}$ such that: (i) if $X$ has type fterm then $v(X) \in H U$, (ii) if $X$ has type state then $v(X) \in \Sigma$, and (iii) if $X$ has type ilist then $v(X) \in \Sigma^{\omega}$. For any term $t$, literal $L$, and clause $\gamma$, we define $v(t), v(L)$, and $v(\gamma)$, by induction on the structure of $t, L$, and $\gamma$, respectively. We will say that $v(t), v(L)$, and $v(\gamma)$, is 'a term', 'a literal', and 'a clause', respectively, also when they are infinite structures.

We extend the notion of Herbrand base [9] to the case of $\omega$-programs by introducing the set $\mathcal{B}_{\omega}$ defined as follows:

$$
\begin{aligned}
\mathcal{B}_{\omega}=\left\{p\left(v\left(X_{1}\right), \ldots, v\left(X_{n}\right)\right) \mid\right. & p \text { is an } n \text {-ary predicate symbol and } \\
v & \text { is a valuation }\}
\end{aligned}
$$

Thus, any $\omega$-interpretation can be identified with a subset of $\mathcal{B}_{\omega}$.

A local stratification is a function $\sigma: \mathcal{B}_{\omega} \rightarrow W$, where $W$ is the set of countable ordinals. Given $A \in \mathcal{B}_{\omega}$, we define $\sigma(\neg A)=\sigma(A)+1$. Given a clause $\gamma$ of the form $H \leftarrow L_{1} \wedge \ldots \wedge L_{m}$ in an $\omega$-program $P$ and a local stratification $\sigma$, we say that $\gamma$ is locally stratified w.r.t. $\sigma$ if for $i=1, \ldots, m$, for every valuation $v$, $\sigma(v(H)) \geq \sigma\left(v\left(L_{i}\right)\right)$. An $\omega$-program $P$ is locally stratified w.r.t. $\sigma$, or $\sigma$ is a local stratification for $P$, if every clause in $P$ is locally stratified w.r.t. $\sigma$. An 
$\omega$-program $P$ is locally stratified if there exists a local stratification $\sigma$ such that $P$ is locally stratified w.r.t. $\sigma$.

Clearly, every stratified $\omega$-program is a locally stratified $\omega$-program. Similarly to the case of logic programs, for every locally stratified $\omega$-program $P$ (and, hence, for every stratified $\omega$-program $P$ ), we can construct a unique perfect $\omega$ model (or perfect model, for short) denoted by $M(P)[1,12]$ (an instance of this construction is presented in Example 1).

Definition 3 (Monadic $\omega$-Programs). A monadic $\omega$-clause is an $\omega$-clause of the form $A_{0} \leftarrow L_{1} \wedge \ldots \wedge L_{m}$, with $m \geq 0$, such that: (i) $A_{0}$ is an atom of the form $p_{0}$ or $q_{0}\left(\left[s \mid X_{0}\right]\right)$, where $q_{0}$ is a predicate of type ilist and $s \in \Sigma$, (ii) for $i=1, \ldots, m, L_{i}$ is either an atom $A_{i}$ or a negated atom $\neg A_{i}$, where $A_{i}$ is of the form $p_{i}$ or $q_{i}\left(X_{i}\right)$, and $q_{i}$ is a predicate of type ilist, and (iii) there exists a level mapping $\ell$ such that, for $i=1, \ldots, m$, if $L_{i}$ is an atom and $\operatorname{vars}\left(A_{0}\right) \nsupseteq \operatorname{vars}\left(L_{i}\right)$, then $\ell\left(A_{0}\right)>\ell\left(L_{i}\right)$ else $\ell\left(A_{0}\right) \geq \ell\left(L_{i}\right)$. A monadic $\omega$-program is a finite set of monadic $\omega$-clauses.

Note that in Definition 3 the predicate symbols $p_{0}, q_{0}, \ldots, p_{m}, q_{m}$ and the variables $X_{0}, \ldots, X_{m}$ are not necessarily distinct. Condition (iii) ensures that a monadic $\omega$-program is stratified. This condition, which is actually stronger than stratification, is also needed for guaranteeing the completeness of the proof method for monadic $\omega$-programs (see Section 3.2).

Example 1. Let $r, q$, and $p$ be predicates of type ilist. The following set of clauses is a monadic $\omega$-program $P$ (and, thus, also an $\omega$-program):

$$
\begin{array}{lll}
p([a \mid X]) \leftarrow p(X) & q([a \mid X]) \leftarrow q(X) & r([a \mid X]) \leftarrow r(X) \\
p([b \mid X]) \leftarrow \neg q(X) & q([a \mid X]) \leftarrow \neg r(X) & r([b \mid X]) \leftarrow \\
& q([b \mid X]) \leftarrow q(X) &
\end{array}
$$

Program $P$ is stratified by the level mapping $\ell:$ Pred $\rightarrow \mathbb{N}$ such that $\ell(p)=2$, $\ell(q)=1$, and $\ell(r)=0$. The perfect model $M(P)$ is constructed starting from the ground atoms of level 0 and going up, level-by-level, as we now indicate. We start from the ground atoms of level 0 , that is, the ground atoms with predicate $r$. For all $w \in\{a, b\}^{\omega}, r(w) \in M(P)$ iff $w \in a^{*} b(a+b)^{\omega}$. Thus, $r(w) \notin M(P)$ iff $w \in a^{\omega}$, that is, $\neg r(w)$ holds in $M(P)$ iff $w \in a^{\omega}$. Then we consider the ground atoms of level 1 , that is, the ground atoms with predicate $q$. For all $w \in\{a, b\}^{\omega}$, $q(w) \in M(P)$ iff $w \in(a+b)^{*} a^{\omega}$ (that is, $w$ has finitely many occurrences of $b$ ). Thus, $\neg q(w)$ holds in $M(P)$ iff $w \in\left(a^{*} b\right)^{\omega}$ (that is, $w$ has infinitely many occurrences of $b$ ). Finally, we consider the ground atoms of level 2, that is, the ground atoms with predicate $p$. For all $w \in\{a, b\}^{\omega}, p(w) \in M(P)$ iff $w \in\left(a^{*} b\right)\left(a^{*} b\right)^{\omega}$, that is, $p(w) \in M(P)$ iff $w \in\left(a^{*} b\right)^{\omega}$.

\subsection{Encoding the CTL $^{*}$ Satisfaction Relation as an $\omega$-Program}

Given a Kripke structure $\mathcal{K}$ and a $\mathrm{CTL}^{*}$ state formula $\varphi$, we introduce a locally stratified $\omega$-program $P_{\mathcal{K}, \varphi}$ which defines, among others, the following three predicates: (i) the unary predicate path such that path $(\pi)$ holds iff $\pi$ is an infinite list 
representing a computation path of $\mathcal{K}$, (ii) the binary predicate sat that encodes the satisfaction relation for $\mathrm{CTL}^{*}$ formulas, in the sense that for all computation paths $\pi$ and $\mathrm{CTL}^{*}$ formulas $\psi$, we have that $M\left(P_{\mathcal{K}, \varphi}\right) \vDash \operatorname{sat}(\pi, \psi)$ iff $\mathcal{K}, \pi \vDash \psi$, and (iii) the nullary predicate prop that encodes the property $\varphi$ to be verified, in the sense that prop holds iff there exists an infinite list $\pi$ whose first element is the initial state $s_{0}$ of $\mathcal{K}$ and $\mathcal{K}, \pi \vDash \varphi$.

When writing terms that encode $\mathrm{CTL}^{*}$ formulas, such as the second argument of the predicate sat, we will use the function symbols $e, x$, and $u$ standing for the operator symbols $\mathrm{E}, \mathrm{X}$, and $\mathrm{U}$, respectively.

Definition 4 (Encoding Program). Given a Kripke structure $\mathcal{K}=\left\langle\Sigma, s_{0}, \rho, \lambda\right\rangle$ and a CTL* formula $\varphi$, the encoding program $P_{\mathcal{K}, \varphi}$ is the following $\omega$-program:

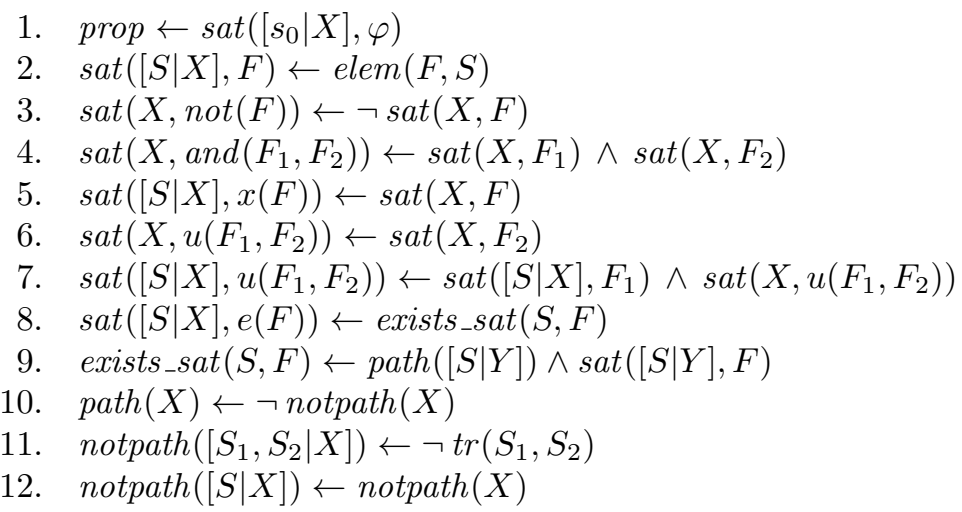

together with the clauses defining the predicates $t r$ and elem, where:

(1) for all states $s_{1}, s_{2} \in \Sigma, \operatorname{tr}\left(s_{1}, s_{2}\right)$ holds iff $\left(s_{1}, s_{2}\right) \in \rho$, and

(2) for every property $d \in$ Elem and state $s \in \Sigma$, elem $(d, s)$ holds iff $d \in \lambda(s)$.

Clause 1 of Definition 4 asserts that the property $\varphi$ holds for an infinite list of states whose first element is $s_{0}$. Clauses $2-9$ define the satisfaction relation $\operatorname{sat}(X, \varphi)$ for any infinite list $X$ and $\mathrm{CTL}^{*}$ formula $\varphi$. The definition of $\operatorname{sat}(X, \varphi)$ is by structural induction on $\varphi$. Clauses 10-12 establish that path $(X)$ holds iff for every pair $\left(a_{i}, a_{i+1}\right)$ of consecutive elements on the infinite list $X$, we have that $\left(a_{i}, a_{i+1}\right) \in \rho$. Indeed, clauses 11 and 12 establish that notpath $(X)$ holds iff in the list $X$ there exist two consecutive elements $a_{i}$ and $a_{i+1}$ such that $\left(a_{i}, a_{i+1}\right) \notin \rho$.

The program $P_{\mathcal{K}, \varphi}$ is locally stratified w.r.t. the stratification function $\sigma$ from ground literals to natural numbers, defined as follows (in what follows, for any $\mathrm{CTL}^{*}$ formula $\chi$, we will denote by $|\chi|$ the number of occurrences of function symbols in $r$ ): for all states $a \in \Sigma$, for all infinite lists $\pi \in \Sigma^{\omega}$, and for all $\mathrm{CTL}^{*}$ formulas $\psi$, (i) $\sigma($ prop $)=|\varphi|+1$, where prop $\leftarrow \operatorname{sat}\left(\left[s_{0} \mid X\right], \varphi\right)$, (ii) $\sigma(\operatorname{sat}(\pi, \psi))=|\psi|+1$, (iii) $\sigma($ exists_sat $(a, \psi))=|\psi|+2$, (iv) $\sigma($ path $(\pi))=2$, (v) $\sigma(\operatorname{notpath}(\pi))=1$, (vi) for every ground atom $A, \sigma(\neg A)=\sigma(A)+1$, and (vii) in all other cases $\sigma$ returns 0 .

Example 2. Let us consider: (i) the set Elem $=\{a, b, t t\}$ of elementary properties, where $t t$ is the elementary property which holds in every state, and 
(ii) the Kripke structure $\mathcal{K}=\left\langle\left\{s_{0}, s_{1}, s_{2}\right\}, s_{0}, \rho, \lambda\right\rangle$, where $\rho$ is the transition relation $\left\{\left(s_{0}, s_{0}\right),\left(s_{0}, s_{1}\right),\left(s_{1}, s_{1}\right),\left(s_{1}, s_{2}\right),\left(s_{2}, s_{1}\right)\right\}$ and $\lambda$ is the function such that $\lambda\left(s_{0}\right)=\{a\}, \lambda\left(s_{1}\right)=\{b\}$, and $\lambda\left(s_{2}\right)=\{a\}$. Let us also consider the formula $\varphi=\mathrm{E}(a \mathrm{U} \neg \mathrm{E}(t t \mathrm{U} \neg(t t \mathrm{U} b)))$, which can be abbreviated as $\mathrm{E}(a \mathrm{U}$ AGF $b)$, where: (i) for every state formula $\psi, \mathrm{F} \psi$ (read 'eventually $\psi$ ') stands for $t t \mathrm{U} \psi$, and $\mathbf{G} \psi$ (read 'always $\psi$ ') stands for $\neg \mathrm{F} \neg \psi$, and (ii) for every path formula $\psi$, $\mathrm{A} \psi$ (read 'for all computation paths $\psi$ ') stands for $\neg \mathrm{E} \neg \psi$. The encoding program $P_{\mathcal{K}, \varphi}$ is as follows:

$$
\begin{aligned}
& \text { prop } \leftarrow \operatorname{sat}\left(\left[s_{0} \mid X\right], e(u(a, \operatorname{not}(e(u(t t, \operatorname{not}(u(t t, b)))))))\right) \\
& \operatorname{tr}\left(s_{0}, s_{0}\right) \leftarrow \operatorname{tr}\left(s_{0}, s_{1}\right) \leftarrow \quad \operatorname{tr}\left(s_{1}, s_{1}\right) \leftarrow \quad \operatorname{tr}\left(s_{1}, s_{2}\right) \leftarrow \quad \operatorname{tr}\left(s_{2}, s_{1}\right) \leftarrow \\
& \operatorname{elem}\left(a, s_{0}\right) \leftarrow \quad \operatorname{elem}\left(b, s_{1}\right) \leftarrow \quad \operatorname{elem}\left(a, s_{2}\right) \leftarrow \quad \operatorname{elem}(t t, S) \leftarrow
\end{aligned}
$$

together with clauses $2-12$ of Definition 4 defining the predicates sat, path, and notpath.

Since $\mathcal{K} \vDash \varphi$ holds iff there exists an infinite list $\pi \in \Sigma^{\omega}$ such that the first state of $\pi$ is the initial state $s_{0}$ of $\mathcal{K}$ and $\mathcal{K}, \pi \vDash \varphi$ holds (see Definition 2), we have that the correctness of $P_{\mathcal{K}, \varphi}$ can be expressed by stating that $\mathcal{K} \vDash \varphi$ holds iff $M\left(P_{\mathcal{K}, \varphi}\right) \vDash \exists X \operatorname{sat}\left(\left[s_{0} \mid X\right], \varphi\right)$ iff (by clause 1 of Definition 4) $M\left(P_{\mathcal{K}, \varphi}\right) \vDash$ prop. The correctness of $P_{\mathcal{K}, \varphi}$ is stated in the following theorem.

Theorem 1 (Correctness of the Encoding Program). Let $P_{\mathcal{K}, \varphi}$ be the encoding program for a Kripke structure $\mathcal{K}$ and a state formula $\varphi$. Then, $\mathcal{K} \vDash \varphi$ iff $M\left(P_{\mathcal{K}, \varphi}\right) \vDash$ prop.

\section{Transformational CTL* Model Checking}

In this section we present a technique based on program transformation for checking whether or not, for any given structure $\mathcal{K}$ and state formula $\varphi, M\left(P_{\mathcal{K}, \varphi}\right) \vDash$ prop holds, where $P_{\mathcal{K}, \varphi}$ is constructed as indicated in Definition 4 above. Our technique consists of two steps. In the first step we transform the $\omega$-program $P_{\mathcal{K}, \varphi}$ into a monadic $\omega$-program $T$ such that $M\left(P_{\mathcal{K}, \varphi}\right) \vDash$ prop iff $M(T) \vDash$ prop. In the second step we check whether or not $M(T) \vDash$ prop holds by using a proof method for monadic $\omega$-programs.

\subsection{Transformation to Monadic $\omega$-Programs}

The first step of our model checking technique is realized by applying specialized versions of the following transformation rules: definition introduction and elimination, instantiation, positive and negative unfolding, clause deletion, positive and negative folding (see, for instance, $[5,14,15]$ ). These rules are applied according to a strategy which is a variant of the specialization strategy presented in $[5]$.

Our specialization strategy starts off from the clause $\gamma_{1}$ : prop $\leftarrow \operatorname{sat}\left(\left[s_{0} \mid X\right], \varphi\right)$ in $P_{\mathcal{K}, \varphi}$ (see clause 1 in Definition 4) and a set of clauses, called InDefs which is initialized to $\left\{\gamma_{1}\right\}$. Then, our strategy iteratively applies two procedures: (i) the 
instantiate-unfold procedure, and (ii) the define-fold procedure. At each iteration, the set InDefs is transformed into a set $D s$ of monadic $\omega$-clauses, at the expense of possibly introducing some auxiliary, non-monadic clauses which are stored in the set NewDefs. These auxiliary clauses are given as input to a subsequent iteration of the strategy. The strategy terminates when no new auxiliary clauses are introduced and, when this happens, in a final step we apply the definition elimination rule by keeping only the clauses whose head predicate is either prop or a predicate on which prop depends.

\section{The Specialization Strategy.}

Input: An $\omega$-program $P_{\mathcal{K}, \varphi}$ for a Kripke structure $\mathcal{K}$ and a state formula $\varphi$. Output: A monadic $\omega$-program $T$ such that $M\left(P_{\mathcal{K}, \varphi}\right) \vDash$ prop iff $M(T) \vDash$ prop.

$Q:=P_{\mathcal{K}, \varphi} ; \quad$ InDefs $:=\left\{\operatorname{prop} \leftarrow \operatorname{sat}\left(\left[s_{0} \mid X\right], \varphi\right)\right\} ; \quad$ Defs $:=$ InDefs;

while InDefs $\neq \emptyset$ do

instantiate-unfold $(Q$, InDefs, $C s)$;

define-fold (Cs, Defs, NewDefs, Ds);

$Q:=(Q-I n D e f s) \cup N e w D e f s \cup D s ;$

InDefs $:=$ NewDefs; $\quad$ Defs $:=$ Defs $\cup$ NewDefs

od;

$T:=\{\gamma \mid \gamma \in Q$ and the head predicate of $\gamma$ is either prop or a predicate on which prop depends\}.

Let us now introduce two notions which are needed for presenting the instantiateunfold and the define-fold procedures. A definition clause is a non-monadic $\omega$-clause of the form $H \leftarrow A$ where: (1) $H$ is an atom of the form $p$ or $q(X)$, where $q$ is a predicate of type ilist, (2) $A$ is an atom, and (3) $\operatorname{vars}(H)=$ $\operatorname{vars}(A)$. A quasi-monadic clause is an $\omega$-clause of the form $H \leftarrow L_{1} \wedge \ldots \wedge L_{k}$, with $k \geq 0$, such that: (i) $H$ is an atom of the form $p$ or $q([s \mid X])$, where $p$ is a predicate of type ilist and $s \in \Sigma$, and (ii) for $i=1, \ldots, k$, there exists a variable $Y$ (possibly equal to $X$ ) of type ilist such that $\operatorname{vars}\left(L_{i}\right) \subseteq\{Y\}$.

The instantiate-unfold procedure transforms a given set InDefs of definition clauses into a set $C s$ of quasi-monadic clauses by: (1) instantiating each clause in InDefs, (2) applying the positive (or negative) unfolding rule to clauses of the form $p([s \mid X]) \leftarrow B_{L} \wedge L \wedge B_{R}$, whenever $L$ is a positive literal (or a negative literal, respectively), and (3) deleting subsumed clauses.

Given a clause $\delta$, a variable $X$, and a term $t$, we denote by $\delta\{X / t\}$ the clause $\delta$ with every occurrence of $X$ replaced by $t$.

The instantiate-unfold Procedure.

Input: An $\omega$-program $Q$ and a set InDefs $\subseteq Q$ of definition clauses.

Output: A set $C s$ of quasi-monadic clauses.

(Instantiation)

Let $Y$ be a new variable of type ilist and let $\Sigma$ be the set of states of $\mathcal{K}$;

$S:=\{\delta\{X /[s \mid Y]\} \mid \delta \in$ InDefs and $\operatorname{vars}(\delta)=\{X\}$ and $s \in \Sigma\} \cup$

$\{\delta \mid \delta \in \operatorname{InDefs}$ and $\operatorname{vars}(\delta)=\emptyset\}$; 
$C s:=\emptyset$;

(Unfolding)

while there exists a clause $\gamma$ in $S$ do

(Case 1. Positive Unfolding)

if $\quad$ (i) $\gamma$ is of the form $H \leftarrow B_{L} \wedge A \wedge B_{R}$, where $A$ is an atom,

(ii) $K_{1} \leftarrow B_{1}, \ldots, K_{m} \leftarrow B_{m}$ are all clauses in $P_{\mathcal{K}, \varphi}$ such that $A$ is unifiable with $K_{1}, \ldots, K_{m}$ with most general unifiers $\vartheta_{1}, \ldots, \vartheta_{m}$, and

(iii) for $i=1, \ldots, m, A=K_{i} \vartheta_{i}$ (that is, $A$ is an instance of $K_{i}$ )

then $S:=(S-\{\gamma\}) \cup\left\{H \leftarrow B_{L} \wedge B_{1} \vartheta_{1} \wedge B_{R}, \ldots, H \leftarrow B_{L} \wedge B_{m} \vartheta_{m} \wedge B_{R}\right\}$

(Case 2. Negative Unfolding)

elseif (i) $\gamma$ is of the form $H \leftarrow B_{L} \wedge \neg A \wedge B_{R}$, where $A$ is an atom,

(ii) $K_{1} \leftarrow B_{1}, \ldots, K_{m} \leftarrow B_{m}$ are all clauses in $P_{\mathcal{K}, \varphi}$ such that $A$ is unifiable with $K_{1}, \ldots, K_{m}$ with most general unifiers $\vartheta_{1}, \ldots, \vartheta_{m}$,

(iii) for $i=1, \ldots, m, A=K_{i} \vartheta_{i}$ (that is, $A$ is an instance of $K_{i}$ ), and

(iv) for $i=1, \ldots, m, \operatorname{vars}\left(B_{i}\right) \subseteq \operatorname{vars}\left(K_{i}\right)$

then from $B_{L} \wedge \neg\left(B_{1} \vartheta_{1} \vee \ldots \vee B_{m} \vartheta_{m}\right) \wedge B_{R}$ we get an equivalent disjunction $Q_{1} \vee \ldots \vee Q_{r}$ of conjunctions of literals, with $r \geq 0$, by first pushing $\neg$ inside and then pushing $\vee$ outside;

$S:=(S-\{\gamma\}) \cup\left\{H \leftarrow Q_{1}, \ldots, H \leftarrow Q_{r}\right\}$

(Case 3. No Unfolding)

else $\quad S:=S-\{\gamma\} ; \quad C s:=C s \cup\{\gamma\} \quad$ fi

od;

(Subsumption)

while there exists a unit clause $\gamma_{1}$ in $C s$ of the form $H \leftarrow$ and a variant of a clause $\gamma_{2}$ in $C s-\left\{\gamma_{1}\right\}$ of the form $H \leftarrow B$ do $C s:=C s-\left\{\gamma_{2}\right\}$ od

The define-fold procedure transforms the quasi-monadic $\omega$-clauses of $C s$ into monadic $\omega$-clauses by applying the definition introduction rule and the (positive or negative) folding rule. In particular, for any given quasi-monadic clause $\gamma$ : $H \leftarrow L_{1} \wedge \ldots \wedge L_{k}$ in $C s$ and for $i=1, \ldots, m$, the define-fold procedure performs the following steps.

Let $L_{i}$ be either the positive literal $A_{i}$ or the negative literal $\neg A_{i}$. We consider the following two cases. Case (1): If in Defs $\cup$ NewDefs there is a clause $\delta_{i}$ of the form $K_{i} \leftarrow A_{i}$, then $\gamma$ is folded using $\delta_{i}$, that is, the occurrence of $L_{i}$ in the body of $\gamma$ is replaced either (i) by $K_{i}$, if $L_{i}=A_{i}$ (positive folding), or (ii) by $\neg K_{i}$, if $L_{i}=\neg A_{i}$ (negative folding). Case (2): Otherwise, if in Defs $\cup$ NewDefs there is no clause of the form $K_{i} \leftarrow A_{i}$, then the definition clause $\delta_{i}: K_{i} \leftarrow A_{i}$, where $K_{i}$ has a new predicate symbol newp ${ }_{i}$, is added to NewDefs (by applying the definition introduction rule). Then, clause $\gamma$ is folded using the newly introduced clause $\delta_{i}$ as in Case (1).

The clause $H \leftarrow M_{1} \wedge \ldots \wedge M_{k}$ derived by folding $\gamma$ using clauses $\delta_{1}, \ldots, \delta_{k}$ is a monadic $\omega$-clause. Indeed, we have that: (1) $H$ is either of the form $p$ or of the form $q([s \mid X])$ (because $\gamma$ is quasi-monadic), (2) for $i=1, \ldots, k, M_{i}$ is either the atom $K_{i}$ or the negated atom $\neg K_{i}$, where $K_{i}$ is either of the form $n e w p_{i}$ or of the 
form newp $p_{i}(Y)$ (this follows from the definition of $\delta_{i}$ and the fact that $\gamma$ is quasimonadic), and (3) Condition (iii) of Definition 3 holds by defining $\ell$ as follows: let $\sigma$ be the stratification function for the encoding program $P_{\mathcal{K}, \varphi}$ (see Section 2.2), (i) $\ell($ prop $)=\sigma($ prop $)=|\varphi|+1$, and (ii) for every predicate newp $i$ that occurs in the head of a clause $K_{i} \leftarrow A_{i}$ introduced during any execution of the definefold procedure, $\ell\left(\right.$ newp $\left.p_{i}\right)=\sigma\left(A_{i}^{\prime}\right)$, where $A_{i}^{\prime}$ is any ground instance of $A_{i}$. For example, if we introduce the definition clause newp $p_{i}(X) \leftarrow \operatorname{sat}(X, e(u(a, b)))$, then we define $\ell\left(\right.$ newp $\left.\left._{i}\right)=\sigma(\operatorname{sat}(\pi, e(u(a, b))))=\mid e(u(a, b))\right) \mid+1=5$, where $\pi$ is any infinite list. Note that $\ell$ does not depend on the particular instance of $A_{i}$, because the value of $\sigma$ is independent of the infinite list which (possibly) occurs as an argument of $A_{i}$.

The define-fold Procedure.

Input: (i) A set Cs of quasi-monadic clauses and (ii) a set Defs of definition clauses;

Output: (i) A set NewDefs of definition clauses, and (ii) a set Ds of monadic $\omega$-clauses.

NewDefs $:=\emptyset ; \quad$ Ds $:=\emptyset ;$

for each clause $\gamma$ in $C s$ do

let the clause $\gamma$ be of the form $H \leftarrow L_{1} \wedge \ldots \wedge L_{k}$;

for $i=1, \ldots, k$ do

let $L_{i}$ be either $A_{i}$ or $\neg A_{i}$, for some atom $A_{i}$;

(Definition Introduction)

if a clause $\delta$ with body $A_{i}$ has a variant in Defs $\cup$ NewDefs

then take $K_{i}$ to be the head of $\delta$

else take $K_{i}$ to be: (i) newp ${ }_{i}(Y)$, if $\operatorname{vars}\left(A_{i}\right)=\{Y\}$, and (ii) newp $p_{i}$, if $\operatorname{vars}\left(A_{i}\right)=\emptyset$, where newp $p_{i}$ is a new predicate symbol; NewDefs $:=$ NewDefs $\cup\left\{K_{i} \leftarrow A_{i}\right\}$ fi;

(Positive or Negative Folding)

if $L_{i}$ is $A_{i}$ then $M_{i}:=K_{i}$ else $M_{i}:=\neg K_{i} \mathbf{f i}$

od

od; $D s:=D s \cup\left\{H \leftarrow M_{1} \wedge \ldots \wedge M_{k}\right\}$

The specialization strategy, which from the initial program $P_{\mathcal{K}, \varphi}$ produces the final program $T$, is correct w.r.t. the perfect model semantics, in the sense that $M\left(P_{\mathcal{K}, \varphi}\right) \vDash$ prop iff $M(T) \vDash p r o p$. This correctness result can be proved similarly to $[5,14]$. Note that the instantiation rule that we use in the unfold procedure, is not present in $[5,14]$, but its application can be viewed as an unfolding of an additional atom ilist $(X)$ defined by the clauses: $\operatorname{ilist}\left(\left[s_{0} \mid Y\right]\right) \leftarrow$, $\ldots$, ilist $\left(\left[s_{h} \mid Y\right]\right) \leftarrow$, where $\Sigma=\left\{s_{0}, \ldots, s_{h}\right\}$ is the set of states of $\mathcal{K}$.

Our specialization strategy terminates for every input program $P_{\mathcal{K}, \varphi}$ because: (i) both the instantiate-unfold and define-fold procedures terminate, and (ii) the while loop of the strategy terminates.

The termination of the instantiate-unfold procedure is a consequence from the following properties. (1) The Instantiation and Subsumption steps terminate. 
(2) The predicates path, tr, and elem do not depend on themselves in program $P_{\mathcal{K}, \varphi}$. (3) For each clause in $P_{\mathcal{K}, \varphi}$ defining the predicate notpath, either the predicate of the body literal does not depend on notpath (see clause 11) or the term occurring in the body is a proper subterm of the term occurring in the head (see clause 12). (4) For each clause in $P_{\mathcal{K}, \varphi}$ whose head is of the form $\operatorname{sat}\left(l_{1}, \psi_{1}\right)$ and for each literal of the form $\operatorname{sat}\left(l_{2}, \psi_{2}\right)$ occurring (positively or negatively) in the body of that clause, either $\psi_{2}$ is a proper subterm of $\psi_{1}$ or $\psi_{1}=\psi_{2}$ and $l_{2}$ is a proper subterm of $l_{1}$. (5) For each state $s$ and formula $\psi$, the literal esists_sat $(s, \psi)$ depends on itself through a call to the predicate sat (see clauses 8 and 9) and by consuming at least one operator $e$ in the formula $\psi$. (6) The applicability conditions given in the instantiate-unfold procedure (see Point (iii) of Case 1 and Case 2) do not allow the unfolding of a clause $\gamma$ if this unfolding instantiates a variable in $\gamma$.

The termination of the define-fold procedure is straightforward.

Finally, the proof of termination of the while loop of the specialization strategy follows from the fact that only a finite number of definition clauses can be introduced by the define-fold procedure. Indeed, every definition clause is of the form $H \leftarrow A$, where: (i) $A$ is an atom in the finite set $\Delta=\{$ notpath $([s \mid X]) \mid$ $s \in \Sigma\} \cup\{$ exists_sat $(s, \psi) \mid s \in \Sigma$ and $\psi$ is a subformula of $\varphi\} \cup\{\operatorname{sat}(X, \psi) \mid \psi$ is a subformula of $\varphi\}$, and (ii) for any $A \in \Delta$ the define-fold procedure introduces at most one definition clause.

Theorem 2 (Correctness and Termination of the Specialization Strategy). Let $P_{\mathcal{K}, \varphi}$ be the encoding program for a Kripke structure $\mathcal{K}$ and a state formula $\varphi$. The specialization strategy terminates for the input program $P_{\mathcal{K}, \varphi}$ and returns an output program $T$ such that: (i) $T$ is a monadic $\omega$-program and (ii) $M\left(P_{\mathcal{K}, \varphi}\right) \vDash$ prop iff $M(T) \vDash$ prop.

Example 3. Let us consider program $P_{\mathcal{K}, \varphi}$ of Example 2. Our specialization strategy starts off from the sets $Q=P_{\mathcal{K}, \varphi}$ and InDefs $=$ Defs $=\left\{\gamma_{1}\right\}$, where $\gamma_{1}$ is the following definition clause (that is, clause 1 of $P_{\mathcal{K}, \varphi}$ ):

$$
\gamma_{1}: \quad \operatorname{prop} \leftarrow \operatorname{sat}\left(\left[s_{0} \mid X\right], e(u(a, \operatorname{not}(e(u(t t, \operatorname{not}(u(t t, b)))))))\right)
$$

In the first execution of the loop body of our strategy we apply the instantiateunfold procedure to the set InDefs. We get the set $C s=\left\{\gamma_{2}, \gamma_{3}\right\}$ of quasimonadic clauses, where:

$$
\begin{aligned}
& \gamma_{2}: \quad \text { prop } \leftarrow \neg \text { notpath }\left(\left[s_{0} \mid X\right]\right) \wedge \operatorname{sat}(X, u(a, \operatorname{not}(e(u(t t, \operatorname{not}(u(t t, b))))))) \\
& \gamma_{3}: \quad \text { prop } \leftarrow \neg \text { notpath }\left(\left[s_{0} \mid X\right]\right) \wedge \neg \text { exists_sat }\left(s_{0}, u(t t, \operatorname{not}(u(t t, b)))\right)
\end{aligned}
$$

Then, by applying the define-fold procedure, we get the set NewDefs $=\left\{\gamma_{4}, \gamma_{5}, \gamma_{6}\right\}$ of definition clauses and the set $D s=\left\{\gamma_{2}^{\prime}, \gamma_{3}^{\prime}\right\}$ of monadic $\omega$-clauses, where:

$$
\begin{array}{ll}
\gamma_{4}: & p_{1}(X) \leftarrow \operatorname{notpath}\left(\left[s_{0} \mid X\right]\right) \\
\gamma_{5}: & p_{2}(X) \leftarrow \operatorname{sat}(X, u(a, \operatorname{not}(e(u(t t, \operatorname{not}(u(t t, b))))))) \\
\gamma_{6}: & p_{3} \leftarrow \text { exists_sat }\left(s_{0}, u(t t, \operatorname{not}(u(t t, b)))\right) \\
\gamma_{2}^{\prime}: & \text { prop } \leftarrow \neg p_{1}(X) \wedge p_{2}(X) \\
\gamma_{3}^{\prime}: & \text { prop } \leftarrow \neg p_{1}(X) \wedge \neg p_{3}
\end{array}
$$


At the end of the first execution of the body of the while loop of our strategy, we get: $Q=\left(P_{\mathcal{K}, \varphi}-\left\{\gamma_{1}\right\}\right) \cup\left\{\gamma_{2}^{\prime}, \gamma_{3}^{\prime}\right\}$, InDefs $=\left\{\gamma_{4}, \gamma_{5}, \gamma_{6}\right\}$, and Defs $=$ $\left\{\gamma_{1}\right\} \cup\left\{\gamma_{4}, \gamma_{5}, \gamma_{6}\right\}$. Since InDefs $\neq \emptyset$ the execution of the while loop continues. After a few more executions of the loop body, the define-fold procedure does not introduce any new clause in NewDefs. Thus, we get InDefs $=\emptyset$ and we derive the final program $Q$. By keeping every clause in $Q$ whose head predicate is either prop or a predicate on which prop depends, we get the following monadic $\omega$-program $T$ :

$\begin{array}{lll}\text { prop } \leftarrow \neg p_{1}(X) \wedge p_{2}(X) & p_{3} \leftarrow \neg p_{1}(X) \wedge \neg p_{7}(X) & p_{7}\left(\left[s_{2} \mid X\right]\right) \leftarrow p_{7}(X) \\ \text { prop } \leftarrow \neg p_{1}(X) \wedge \neg p_{3} & p_{3} \leftarrow \neg p_{1}(X) \wedge p_{8}(X) & p_{8}\left(\left[s_{0} \mid X\right]\right) \leftarrow \neg p_{7}(X) \\ p_{1}\left(\left[s_{0} \mid X\right]\right) \leftarrow p_{1}(X) & p_{4}\left(\left[s_{0} \mid X\right]\right) \leftarrow & p_{8}\left(\left[s_{0} \mid X\right]\right) \leftarrow p_{8}(X) \\ p_{1}\left(\left[s_{1} \mid X\right]\right) \leftarrow p_{4}(X) & p_{4}\left(\left[s_{1} \mid X\right]\right) \leftarrow p_{4}(X) & p_{8}\left(\left[s_{1} \mid X\right]\right) \leftarrow p_{8}(X) \\ p_{1}\left(\left[s_{2} \mid X\right]\right) \leftarrow & p_{4}\left(\left[s_{2} \mid X\right]\right) \leftarrow p_{9}(X) & p_{8}\left(\left[s_{2} \mid X\right]\right) \leftarrow \neg p_{7}(X) \\ p_{2}\left(\left[s_{0} \mid X\right]\right) \leftarrow \neg p_{3} & p_{5} \leftarrow \neg p_{4}(X) \wedge p_{8}(X) & p_{8}\left(\left[s_{2} \mid X\right]\right) \leftarrow p_{8}(X) \\ p_{2}\left(\left[s_{0} \mid X\right]\right) \leftarrow p_{2}(X) & p_{6} \leftarrow \neg p_{9}(X) \wedge \neg p_{7}(X) & p_{9}\left(\left[s_{0} \mid X\right]\right) \leftarrow \\ p_{2}\left(\left[s_{1} \mid X\right]\right) \leftarrow \neg p_{5} & p_{6} \leftarrow \neg p_{9}(X) \wedge p_{8}(X) & p_{9}\left(\left[s_{1} \mid X\right]\right) \leftarrow p_{4}(X) \\ p_{2}\left(\left[s_{2} \mid X\right]\right) \leftarrow \neg p_{6} & p_{7}\left(\left[s_{0} \mid X\right]\right) \leftarrow p_{7}(X) & p_{9}\left(\left[s_{2} \mid X\right]\right) \leftarrow \\ p_{2}\left(\left[s_{2} \mid X\right]\right) \leftarrow p_{2}(X) & p_{7}\left(\left[s_{1} \mid X\right]\right) \leftarrow & \end{array}$

\subsection{A Proof Method for Monadic $\omega$-Programs.}

In this section we present the second step of our model checking technique. In particular, we present a method for checking whether or not $M(P) \vDash F$ holds, for any monadic $\omega$-program $P$ and any formula $F$ which is either of the form $p$ or of the form $\exists X\left(L_{1} \wedge \ldots \wedge L_{n}\right)$, with $n \geq 1$, where, for $i=1, \ldots, n, L_{i}$ is either a positive literal $q_{i}(X)$ or a negative literal $\neg q_{i}(X)$. In what follows the set of the formulas $F$ of this form will be denoted by $\mathcal{F}$. In particular, our method allows us to check whether or not $M(T) \vDash$ prop holds for the monadic $\omega$-program $T$ that we derive by the specialization strategy presented in Section 3.1.

First, we introduce the notion of a derivation tree and, then, the notion of a proof of a formula $F$ in $\mathcal{F}$ w.r.t. a monadic $\omega$-program $P$. Every node of a derivation tree has: (i) a depth which is the number of its ancestor nodes (in particular, the root has depth 0), and (ii) a label which is either

(1) the empty conjunction true, or

(2) the empty disjunction false, or

(3) a literal of the form: either $p$, or $\neg p$, or $q(X)$, or $\neg q(X)$, or

(4) a formula of the form: either $\exists X\left(L_{1} \wedge \ldots \wedge L_{n}\right)$ or $\neg \exists X\left(L_{1} \wedge \ldots \wedge L_{n}\right)$, with $n \geq 1$, where, for $i=1, \ldots, n, L_{i}$ is either $q_{i}(X)$ or $\neg q_{i}(X)$.

We denote by $\mathcal{L}$ the set of formulas of the forms (3) and (4). Let us also introduce the following notation: (i) for any atom $A, \bar{A}$ denotes $\neg A$ and $\overline{\neg A}$ denotes $A$, and (ii) for any formula $B, \overline{\exists X B}$ denotes $\neg \exists X B$.

In order to construct a derivation tree of a formula in $\mathcal{F}$ w.r.t. a given monadic $\omega$-program $P$, we begin by rewriting the program $P$ as follows. (Recall that in the body of a monadic $\omega$-clause at most one variable occurs in a literal and two distinct literals may have a variable in common.) For every clause $H \leftarrow B$ in $P$ 
and for every variable $Y$ in $\operatorname{vars}(B)-\operatorname{vars}(H)$, we replace the literals $L_{1}, \ldots, L_{m}$ of $B$ such that $\operatorname{vars}\left(L_{1}\right)=\ldots=\operatorname{vars}\left(L_{m}\right)=\{Y\}$ by the formula $\exists Y\left(L_{1} \wedge \ldots \wedge L_{m}\right)$. Thus, every clause in $P$ is rewritten as $H \leftarrow F_{1} \wedge \ldots \wedge F_{k}$, where, for $i=1, \ldots, k$, $F_{i}$ is a formula in $\mathcal{L}$.

For instance, clause $q_{0}([s \mid X]) \leftarrow q_{1}(X) \wedge q_{2}(Y) \wedge p_{1} \wedge \neg q_{3}(Y) \wedge p_{2}$ is rewritten as $q_{0}([s \mid X]) \leftarrow q_{1}(X) \wedge \exists Y\left(q_{2}(Y) \wedge \neg q_{3}(Y)\right) \wedge p_{1} \wedge p_{2}$.

Definition 5 (Derivation Tree). Given a monadic $\omega$-program $P$ and a formula $F$ in $\mathcal{F}$, a derivation tree of $F$ w.r.t. $P$ is a finite tree $T$ constructed as follows:

1. the root node is labeled by $F$, and if $F$ is of the form $\exists X\left(L_{1} \wedge \ldots \wedge L_{n}\right)$ then the root node has $n$ children labeled by $L_{1}, \ldots, L_{n}$, respectively,

2. if a non-root node $N$ is labeled by: (i) true, or (ii) false, or (iii) $\exists X B$, or (iv) $\neg \exists X B$ (that is, $N$ is not labeled by a literal), then $N$ is a leaf,

3. for every integer $d \geq 0$, consider the nodes $N_{1}, \ldots, N_{\ell}$, with $\ell \geq 1$, of depth $d$ : if there exists an integer $c$, with $0 \leq c<d$, such that for every literal $L$ labeling a node of depth $d$, there exists a node of depth $c$ labeled by $L$ then the nodes $N_{1}, \ldots, N_{\ell}$ are leaves

else choose a state $s \in \Sigma$ and, for $i=1, \ldots, \ell$, if the node $N_{i}$ is labeled by a literal $L_{i}$, then construct a child node of $N_{i}$ with label $F$, for each formula $F$ in the set $\mathcal{C}_{i}$ of formulas in $\mathcal{L} \cup\{$ true, false $\}$ constructed from the state $s$, the literal $L_{i}$, and the program $P$, as we now indicate. There are two cases.

Case (i): $L_{i}$ is an atom $q(X)$ (or $p$ ). If in $P$ there is no clause whose head is $q([s \mid X])$ (or $p$ ), then take $\mathcal{C}_{i}$ to be $\{$ false $\}$. Otherwise, choose a clause $q([s \mid X]) \leftarrow F_{1} \wedge \ldots \wedge F_{k}$ (or $p \leftarrow F_{1} \wedge \ldots \wedge F_{k}$ ) in $P$, where, for $i=1, \ldots, k, F_{i} \in \mathcal{L}$. If $k=0$ then take $\mathcal{C}_{i}$ to be $\{$ true $\}$, else take $\mathcal{C}_{i}$ to be $\left\{F_{1}, \ldots, F_{k}\right\}$.

Case (ii): $L_{i}$ is a negated atom $\neg q(X)$ (or $\neg p$ ). Let $q([s \mid X]) \leftarrow B_{1}, \ldots$, $q([s \mid X]) \leftarrow B_{k}$ (or $p \leftarrow B_{1}, \ldots, p \leftarrow B_{k}$ ) be all clauses in $P$ whose head is $q([s \mid X])$ (or $p$ ). If $k=0$ then take $\mathcal{C}_{i}$ to be $\{$ true $\}$. If $k \geq 1$ and there exists $i$, with $1 \leq i \leq k$, such that $B_{i}$ is the empty conjunction, then take $\mathcal{C}_{i}$ to be $\{$ false $\}$. Otherwise, for $i=1, \ldots, k$, choose a formula $F_{i} \in \mathcal{L}$ such that $B_{i}=G_{1} \wedge F_{i} \wedge G_{2}$, where $G_{1}$ and $G_{2}$ are (possibly empty) conjunctions, and take $\mathcal{C}_{i}$ to be $\left\{\bar{F}_{1}, \ldots, \bar{F}_{k}\right\}$.

By construction, for any derivation tree $T$ there exist: (i) an integer $m$ which is the maximal depth of a node of $T$, and (ii) a least integer $c$, with $0 \leq c<m$, such that for every literal $L$ labeling a node of depth $m$, there exists a node of depth $c$ labeled by $L$. Now, we introduce a relation $r_{T}$ between literals as follows. For any two literals $L_{1}$ and $L_{2}, r_{T}\left(L_{1}, L_{2}\right)$ holds iff: (i) there exists a node $M$ of depth $c$ in $T$ whose label is $L_{1}$, (ii) there exists a node $N$ of depth $m$ in $T$ whose label is $L_{2}$, and (iii) $M$ is an ancestor of $N$ in $T$. We denote by $r_{T}^{+}$the transitive closure of $r_{T}$.

Proposition 1. Let $P$ be a monadic $\omega$-program and $F$ be a formula in $\mathcal{F}$. (i) Every derivation tree $T$ of $F$ w.r.t. $P$ is minimal, in the sense that no proper subtree 
of $T$ is itself a derivation tree of $F$ w.r.t. $P$. (ii) There exists a finite number of derivation trees of $F$ w.r.t. $P$.

Now we present the definitions of proof and refutation, which are based on the notion of derivation tree.

Definition 6 (Proof and Refutation). Let $P$ be a monadic $\omega$-program and $F$ be a formula in $\mathcal{F}$. We say that $F$ has a proof w.r.t. $P$ iff there exists a derivation tree $T$ of $F$ w.r.t. $P$ which satisfies the following conditions:

1. every leaf $N$ of $T$ is labeled by: either (i) true, or (ii) a literal of the form $p$, or $\neg p$, or $q(X)$, or $\neg q(X)$, or (iii) a formula of the form $\exists X B$ that has a proof w.r.t. $P$, or (iv) a formula of the form $\neg \exists X B$ such that $\exists X B$ has a refutation w.r.t. $P$,

2. for every positive literal $L$ labeling a leaf of $T, r_{T}^{+}(L, L)$ does not hold.

We say that $F$ has a refutation w.r.t. $P$ iff no derivation tree of $F$ w.r.t. $P$ is a proof of $F$ w.r.t. $P$.

By Proposition 1 it is decidable whether or not a there exists a proof of a formula in $\mathcal{F}$ w.r.t. a monadic $\omega$-program. Moreover, by induction on the level of the predicates occurring in the monadic $\omega$-program $P$, we can show that our proof method is sound and complete for showing that a formula in the set $\mathcal{F}$ is true in the perfect model of $P$. Thus, we have the following result.

Theorem 3. Let $P$ be a monadic $\omega$-program and $F$ a formula in $\mathcal{F}$. Then:

(i) there is an algorithm to check whether or not $F$ has a proof w.r.t. $P$, and

(ii) $F$ has a proof w.r.t. $P$ iff $M(P) \vDash F$.

Now we present an example of application of the second step of our transformational method for proving CTL* properties of the Kripke structures which encode reactive systems.

Example 4. Let us consider: (i) the monadic $\omega$-program $T$, obtained as the output of our specialization strategy (see Example 3), and (ii) the formula prop, that encodes the $\mathrm{CTL}^{*}$ property $\varphi$ of the Kripke structure $\mathcal{K}$ introduced in Example 2. We can construct a proof for the formula prop w.r.t. $T$ as shown by the various derivation trees depicted in Figure 1. As a consequence, we have that $M\left(P_{\mathcal{K}, \varphi}\right) \vDash$ prop holds and, thus, the formula $\varphi$ holds in the Kripke structure $\mathcal{K}$.

\section{Related Work and Concluding Remarks}

Various logic programming techniques and tools have been developed for model checking. In particular, tabled resolution has been shown to be quite effective for implementing a modal $\mu$-calculus model checker for CCS value passing programs [13]. Techniques based on logic programming, constraint solving, abstract interpretation, and program transformation have been proposed for 
(A)

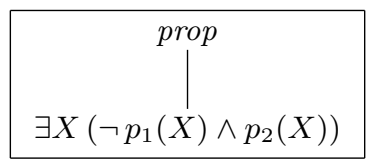

(B)

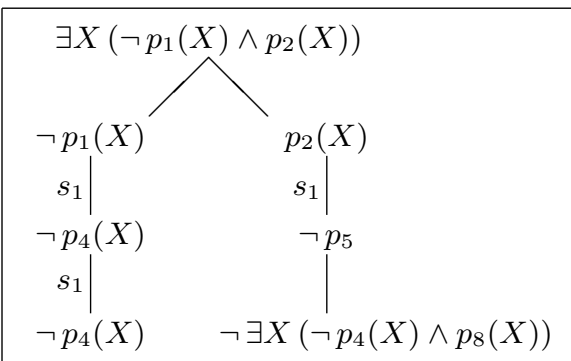

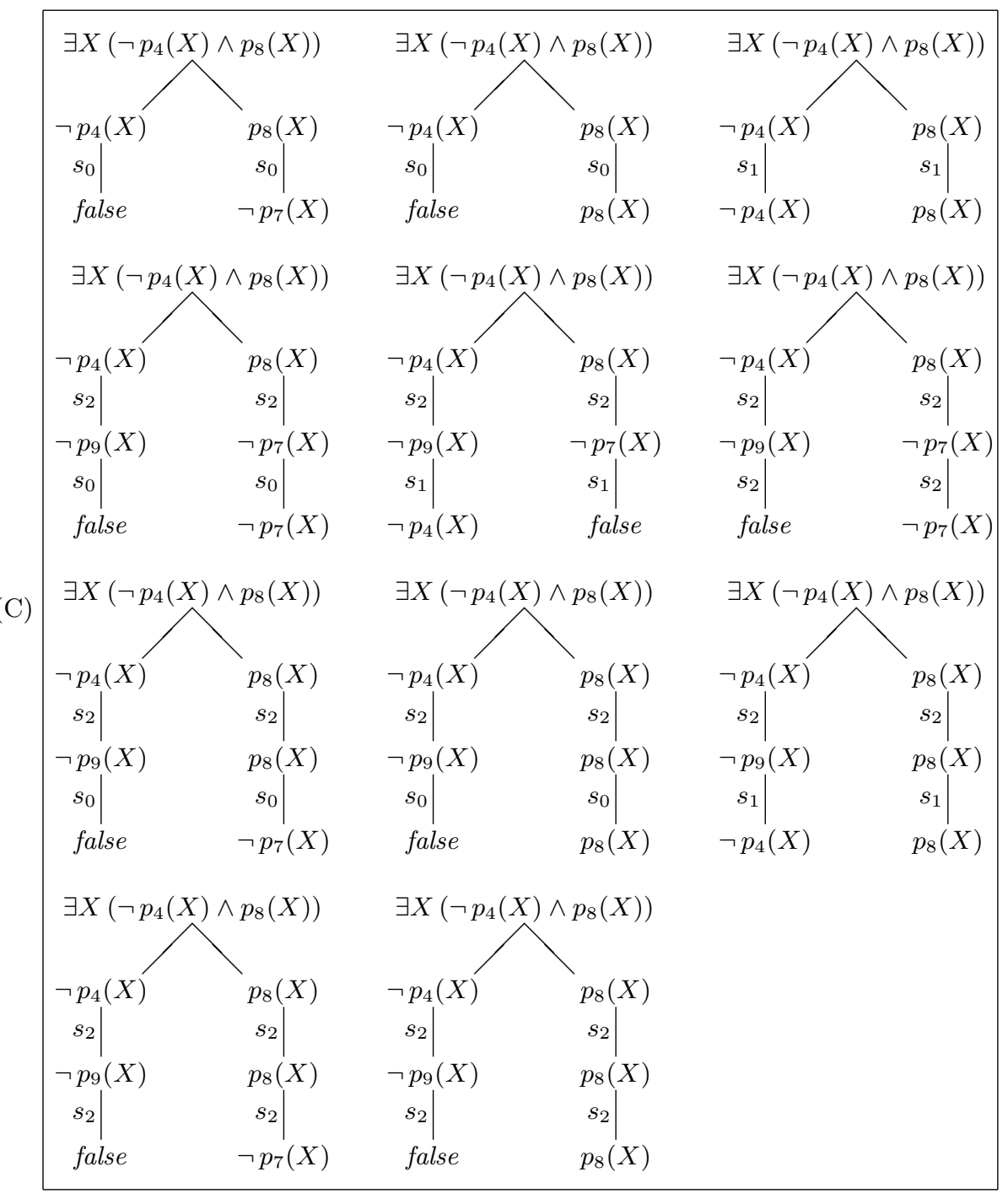

Fig. 1. The tree in (A) is a proof of prop w.r.t. T. The tree in (B) is a proof of $\exists X\left(\neg p_{1}(X) \wedge p_{2}(X)\right)$ w.r.t. $T$. The 11 trees in $(\mathrm{C})$ are all the derivation trees of $\exists X\left(\neg p_{4}(X) \wedge p_{8}(X)\right)$ w.r.t. $T$ and none of them is a proof. The labels of the arcs are the states $s \in \Sigma$ to be chosen according to Point (3) of Definition 5. 
performing CTL model checking of finite and infinite state systems (see, for instance, $[3,5,8,10])$. In this paper we have extended to $\mathrm{CTL}^{*}$ model checking the transformational approach which was proposed for LTL model checking in [11].

The main contributions of this work are the following. (i) We have proposed a method for specifying CTL* properties of reactive systems based on $\omega$-programs, that is, logic programs acting on infinite lists. This method is a proper extension of the methods for specifying CTL or LTL properties, because CTL and LTL are fragments of CTL*. (ii) We have introduced the subclass of monadic $\omega$-programs for which the satisfaction relation w.r.t. the perfect model is decidable. This subclass of programs properly extends the class of linear monadic $\omega$-programs introduced in [11]. (iii) Finally, we have shown that we can transform, by applying semantics preserving unfold/fold rules, the logic programming specification of a $\mathrm{CTL}^{*}$ property into a monadic $\omega$-program.

Our transformation strategy can be viewed as a specialization of the Encoding Program (see Definition 4) w.r.t. a given Kripke structure $\mathcal{K}$ and a given $\mathrm{CTL}^{*}$ formula $\varphi$. However, it should be noted that this program specialization could not be achieved by using partial deduction techniques (see [7] for a brief survey). Indeed, our specialization strategy performs instantiation and negative unfolding steps that cannot be realized by partial deduction.

Our two step verification approach bears some similarities with the automatatheoretic approach to $\mathrm{CTL}^{*}$ model checking, where the specification of a finite state system and a $\mathrm{CTL}^{*}$ formula are translated into alternating tree automata [6]. The automata-theoretic approach is quite appealing because many useful techniques are available in the field of automata theory. However, we believe that also our approach has its advantages because of the following reasons. (1) The specification of properties of reactive systems, together with the transformation of these specifications into monadic $\omega$-programs, and the proofs of properties of monadic $\omega$-programs, can all be done within the single framework of logic programming, while in the automata-theoretic approach one has to translate the temporal logic formalism into the distinct formalism of automata theory. (2) The translation of a specification into a monadic $\omega$-program can be performed by using semantics preserving transformation rules, thereby avoiding the burden of proving the correctness of the translation by ad-hoc methods. (3) Finally, due its generality, we believe that our approach can be extended without much effort to the case of infinite state systems.

Issues that can be investigated in the future include: (i) the complexity of our verification method and, in particular, an efficient implementation of the proof method presented in Section 3.2, (ii) the relationship between monadic $\omega$-programs and alternating tree automata, (iii) the applicability of our transformational approach to other logics, such as the monadic second order logic of successors, and (iv) the experimental evaluation of the efficiency of our transformational approach by considering various test cases and comparing its performance in practical examples w.r.t. that of other model checking techniques known in the literature. 


\section{References}

1. K. R. Apt and R. N. Bol. Logic programming and negation: A survey. Journal of Logic Programming, 19, 20:9-71, 1994.

2. E. M. Clarke, O. Grumberg, and D. Peled. Model Checking. MIT Press, 1999.

3. G. Delzanno and A. Podelski. Constraint-based deductive model checking. International Journal on Software Tools for Technology Transfer, 3(3):250-270, 2001.

4. E. A. Emerson and J. Y. Halpern. "sometimes" and "not never" revisited: on branching versus linear time temporal logic. Journal of the ACM, 33(1):151-178, 1986.

5. F. Fioravanti, A. Pettorossi, and M. Proietti. Verifying CTL properties of infinite state systems by specializing constraint logic programs. In Proceedings of the ACM Sigplan Workshop on Verification and Computational Logic VCL'01, Florence (Italy), Technical Report DSSE-TR-2001-3, pages 85-96. University of Southampton, UK, 2001.

6. O. Kupferman, M. Y. Vardi, and P. Wolper. An automata-theoretic approach to branching-time model checking. Journal of the ACM, 47(2):312-360, 2000.

7. M. Leuschel. Logic program specialisation. In J. Hatcliff and P. Thiemann (Eds.) T. Mogensen, editors, Partial Evaluation - Practice and Theory, Lecture Notes in Computer Science 1706, pages 155-188. Springer, 1998.

8. M. Leuschel and T. Massart. Infinite state model checking by abstract interpretation and program specialization. In A. Bossi, editor, Proceedings of the 9th International Workshop on Logic-based Program Synthesis and Transformation (LOPSTR '99), Venezia, Italy, Lecture Notes in Computer Science 1817, pages 63-82. Springer, 2000.

9. J. W. Lloyd. Foundations of Logic Programming. Springer-Verlag, Berlin, 1987. Second Edition.

10. U. Nilsson and J. Lübcke. Constraint logic programming for local and symbolic model-checking. In J. W. Lloyd, editor, Proceedings of the First International Conference on Computational Logic (CL 2000), London, UK, 24-28 July, Lecture Notes in Artificial Intelligence 1861, pages 384-398. Springer-Verlag, 2000.

11. A. Pettorossi, M. Proietti, and V. Senni. Transformational verification of linear temporal logic. In 24th Italian Conference on Computational Logic June 24-26, 2009, Ferrara, Italy (CILC '09). http://www.ing.unife.it/eventi/cilc09.

12. T. C. Przymusinski. On the declarative semantics of stratified deductive databases and logic programs. In J. Minker, editor, Foundations of Deductive Databases and Logic Programming, pages 193-216. Morgan Kaufmann, 1988.

13. Y. S. Ramakrishna, C. R. Ramakrishnan, I. V. Ramakrishnan, S. A. Smolka, T. Swift, and D. S. Warren. Efficient model checking using tabled resolution. In Proceedings of the 9th International Conference on Computer Aided Verification ( $C A V$ '97), Lecture Notes in Computer Science 1254, pages 143-154. SpringerVerlag, 1997.

14. H. Seki. Unfold/fold transformation of stratified programs. Theoretical Computer Science, 86:107-139, 1991.

15. H. Tamaki and T. Sato. Unfold/fold transformation of logic programs. In S.-A. Tärnlund, editor, Proceedings of the Second International Conference on Logic Programming (ICLP'84), pages 127-138, Uppsala, Sweden, 1984. Uppsala University. 